\title{
ZP2-coated beads decoy sperm
}

Agarose beads with zona pellucida sperm-binding protein 2 (ZP2) attached to them effectively decoy sperm in vitro and in vivo and could offer new methods of non-hormonal contraception and sperm selection for assisted-reproduction technologies, according to new research published in Science Translational Medicine.

The zona pellucida that surrounds ovulated eggs is composed of homologous glycoproteins designated ZP1 to ZP4 and ZP2 is required and sufficient for sperm binding in humans and in mice.

Avella and colleagues created agarose beads coated in either human or mouse $\mathrm{ZP} 2$. In vitro, mouse sperm bound to beads coated with mouse ZP2 but not uncoated beads. In the presence of coated beads, only $6.8 \%$ of inseminated eggs were fertilized after $24 \mathrm{~h}$ compared with $84.5 \%$ of $75.8 \%$ of eggs in the absence of beads or presence of uncoated beads, respectively, were fertilized within the same time period. Freshly isolated or incubated sperm bound to coated beads in comparable numbers, and underwent acrosome exocytosis. Over time, sperm lost motility, membrane integrity and the ability to fertilize eggs once they had bound to coated beads.

Human sperm bound to beads that were coated in human ZP2 in vitro. Freshly isolated or thawed sperm were decoyed by the coated beads in the presence of transgenic mouse eggs expressing human ZP2. Sperm that had bound after 30 minutes of incubation could be detached from the beads by gentle pipetting. Of these initially bound sperm, $62.6 \%$ had intact acrosomes compared with $53.2 \%$ of the starting population. Sperm selection using the coated beads increased the number of sperm that bound to the zona pellucida or penetrated the zona matrix of an egg.

In vivo, coated beads transcervically administered to mice hindered sperm in reaching the oviduct, and embryos were rarely observed (average 1.3 embryos compared with 21.0 in mice treated with medium only or uncoated beads). It took an average of

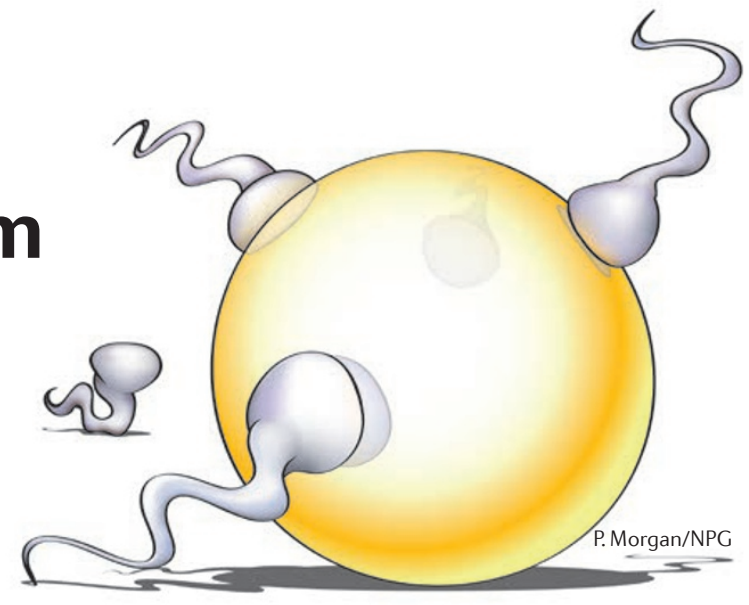

72.8 days after mating for mice treated with coated beads to deliver pups, compared with 28.2 days for mice treated with medium or uncoated beads.

These data suggest that ZP2-coated beads could provide a new form of hormonefree, reversible contraception and that shortterm reversible sperm binding could be an effective method for selecting sperm for use in assisted-reproduction technologies.

Louise Stone

ORIGINAL ARTICLE Avella, M. A. et al. ZP2 peptide beads select human sperm in vitro, decoy mouse sperm in vivo, and provide reversible contraception. Sci. Transl. Med. http:// dx.doi.org/10.1126/scitranslmed.aad9946 (2016). 